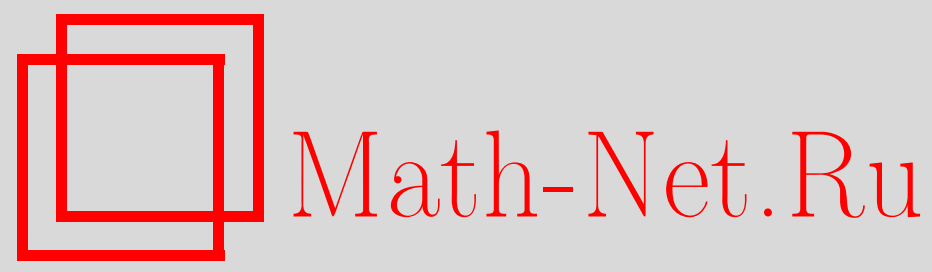

А. А. Туганбаев, Автоморфизмы подмодулей и их продолжения, Дискрет. матем., 2013, том 25, выпуск 1, 144-151

DOI: https://doi.org/10.4213/dm1227

Использование Общероссийского математического портала Math-Net.Ru подразумевает, что вы прочитали и согласны с пользовательским соглашением http://www.mathnet.ru/rus/agreement

Параметры загрузки:

IP: 52.87 .193 .239

26 апреля 2023 г., 17:10:50 


\title{
Автоморфизмы подмодулей и их продолжения
}

\author{
๑) 2013 г. А. А. Туганбаев
}

\begin{abstract}
Исследуются модули, у которых все автоморфизмы подмодулей продолжаются до эндоморфизмов всего модуля.

Работа выполнена при поддержке Российского фонда фундаментальных исследований, проект 08-01-00693-а.
\end{abstract}

Все кольца предполагаются ассоциативными и с ненулевой единицей, а модули унитарными. Модуль называется артиновым, если каждая убывающая цепь его подмодулей стабилизируется на конечном шаге. Выражение вида “ $A$ есть артиново кольцо” означает, что $A_{A}$ и ${ }_{A} A-$ артиновы модули.

Рассмотрим три условия на модуль $M$.

(I) $M-$ квазиинъективный модуль. Напомним, что модуль $M$ называется квазиинъективным, если для любого подмодуля $X$ в $M$ каждый гомоморфизм $X \rightarrow M$ продолжается до эндоморфизма модуля $M$. Хорошо известно, см. например, [7] или [4, Следствие 19.3], что модуль $M$ квазиинъективен в точности тогда, когда $M$ - вполне инвариантный подмодуль своей инъективной оболочки. Напомним, что подмодуль $M$ модуля $E$ называется вполне инвариантным (соответственно, характеристическим), если $f(M) \subseteq M$ для каждого эндоморфизма (соответственно, автоморфизма) $f$ модуля $E$.

(II) $M$ - характеристический подмодуль своей инъективной оболочки. Такие модули изучались, например, в [8, 12] и называются в этих работах автоморфизминвариантными.

(III) $M-$ автоморфизм-продолжаемый модуль, то есть для любого подмодуля $X$ в $M$ каждый автоморфизм модуля $X$ продолжается до эндоморфизма модуля $M$. По утверждению 2 леммы 1 данной работы, модуль $M$ является автоморфизмпродолжаемым в точности тогда, когда для любого существенного подмодуля $X$ в $M$ каждый автоморфизм модуля $X$ продолжается до эндоморфизма модуля $M$. Напомним, что подмодуль $X$ модуля $M$ называется существенным, если $X \cap Y \neq 0$ для любого ненулевого подмодуля $Y$ из $M$.

Заметим, что импликация (I) $\Rightarrow$ (II) очевидна, а импликация (II) $\Rightarrow$ (III) доказана в лемме 3 настоящей работы. В общем случае импликация (III) $\Rightarrow$ (II) неверна. Действительно, аддитивная группа рациональных чисел $\mathbf{Q}$ является инъективной оболочкой модуля $\mathbf{Z}$ над кольцом целых чисел $\mathbf{Z}$. Кроме того, $\mathbf{Z}$ не является характеристическим подмодулем $\mathbf{Z}$-модуля $\mathbf{Q}$, поскольку $\alpha(\mathbf{Z}) \nsubseteq \mathbb{Z}$, где $\alpha: q \rightarrow q / 2$ - автоморфизм $\mathbf{Z}$-модуля 
Q. Тем не менее, непосредственно проверяется, что любой автоморфизм произвольного подмодуля $\mathbf{Z}$-модуля $\mathbf{Z}$ продолжается на $\mathbf{Z}$.

В общем случае импликация (II) $\Rightarrow$ (I) тоже неверна, даже для конечных циклических модулей над конечными алгебрами над полями.

Пример 1. Пусть $F$ - поле порядка $2, A-5$-мерная $F$-алгебра над полем $F$, образованная всеми $(3 \times 3)$-матрицами вида $\left(\begin{array}{ccc}f_{11} & f_{12} & f_{13} \\ 0 & f_{22} & 0 \\ 0 & 0 & f_{33}\end{array}\right)$, где $f_{i j} \in F$. В [12] показано, что

$$
e_{11} A=e_{11} F+e_{12} F+e_{13} F
$$

- конечный циклический автоморфизм-инвариантный модуль, не являющийся квазиинъективным, причем $A$ - конечное полуцепное слева кольцо, не являющееся полуцепным справа. Напомним, что модуль называется цепным, если любые два его подмодуля сравнимы по включению. Прямые суммы цепных модулей называются полуцепными модулями.

Хорошо известно (см., например, [4, 25.4.2]), что каждый модуль над артиновым полуцепным кольцом $A$ является полуцепным модулем. В частности, каждый $A$-модуль является прямой суммой равномерных модулей. Напомним, что модуль называется равномерным, если пересечение любых двух его ненулевых подмодулей не равно нулю. В связи с изложенным выше, мы сформулируем теорему 1, которая применима к конечным полуцепным кольцам и является первым основным результатом данной работы.

Теорема 1. Пусть $A-$ кольцио, над которым каждый правый модуль является прямой суммой равномерных модулей (это так, например, если $A$ - артиново полуцепное кольцо). Для любого правого А-модуля М равносильнь условия:

(1) М - автоморфизм-продолжаемьй модуль;

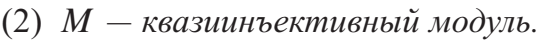

В связи с теоремой 1 заметим, что по изложенному выше теорема 1 неверна для конечных колец, которые являются полуцепными только слева. Кроме того, класс колец, над которыми каждый правый модуль является прямой суммой равномерных модулей, шире класса всех артиновых полуцепных колец, поскольку существуют кольца, над которыми каждый правый модуль является прямой суммой равномерных модулей, но не каждый левый модуль является прямой суммой равномерных модулей. Примером такого кольца является кольцо $A$, образованное всеми $(3 \times 3)$-матрицами вида $\left(\begin{array}{ccc}f_{11} & 0 & 0 \\ f_{21} & f_{22} & 0 \\ f_{31} & 0 & f_{33}\end{array}\right)$, где все $f_{i j}$
- элементы произвольного поля $F$.

Модуль $M$ называется псевдоинъективным, если для любого подмодуля $X$ в $M$ каждый мономорфизм $f$ из $X$ в $M$ продолжается до эндоморфизма модуля $M$ (см. $[10,6,14])$.

Ясно, что каждый квазиинъективный модуль псевдоинъективен. В $[6,14]$ приведены примеры, показывающие, что существуют псевдоинъективные модули, не являющиеся квазиинъективными. Каждый псевдоинъективный модуль автоморфизм-инвариантен (см. лемму 2 ниже). По лемме 3 данной работы, в каждом автоморфизм-инвариантном модуле $M$ выполняется условие III: для любого существенного подмодуля $X$ в $M$ каждый автоморфизм модуля $X$ продолжается до эндоморфизма модуля $M$. Модуль $\mathbf{Z}_{\mathbf{Z}}$ удовлетворяет условию III, но не автоморфизм-инвариантен; в частности, $M$ не псевдоинъективен.

В связи с изложенным выше мы сформулируем теорему 2, которая применима к конечным модулям и является вторым основным результатом данной работы.

Теорема 2. Если $М$ - артинов модуль, то равносильны условия: 
(1) М-автоморфизм-продолжаемый модуль;

(2) $М-$ псевдоинъективный модуль.

Мы разобьем доказательство теорем 1 и 2 на ряд лемм.

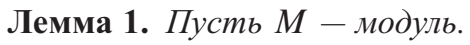

(1) Для любого подмодуля $X^{\prime}$ в $M$ каждый эндоморфизм (соответственно, автоморфизм) модуля $X^{\prime}$ продолжается до эндоморфизма (соответственно, автоморфизма) некоторого существенного подмодуля $X$ в $M$.

(2) Модуль М является автоморфизм-продолжаемым в точности тогда, когда для любого существенного подмодуля $X$ в $M$ каждый автоморфизм модуля $X$ продолжается до эндоморфизма модуля $M$.

(3) Все прямые слагаемые автоморфизм-продолжаемых модулей являются автоморфизм-продолжаемыми модулями.

Доказательство. Докажем первое утверждение. Пусть $\alpha^{\prime}-$ эндоморфизм (соответственно, автоморфизм) модуля $X^{\prime}, Y$ - подмодуль в $M$, максимальный среди подмодулей модуля $M$, имеющих нулевое пересечение с $X^{\prime}$. Тогда

$$
X=X^{\prime} \oplus Y
$$

- существенный подмодуль в $M$. Теперь можно определить эндоморфизм (соответственно, автоморфизм) $\alpha$ модуля $X$ так, что

$$
\alpha\left(x^{\prime}+y\right)=\alpha\left(x^{\prime}\right)+y
$$

для любых $x^{\prime} \in X^{\prime}$ и $y \in Y$.

Второе утверждение вытекает из первого.

Третье утверждение проверяется непосредственно.

Лемма 2. Для модуля М равносильнь условия:

(1) М-автоморфизм-инвариантный модуль;

(2) каждый изоморфизм между любыми существенными подмодулями модуля М продолжается до эндоморфизма модуля $M$.

Следовательно, каждый псевдоинъективный модуль автоморфизм-инвариантен.

Доказательство. Пусть $E-$ инъективная оболочка модуля $M$.

Докажем, что (1) $\Rightarrow$ (2). Пусть $X$ и $X^{\prime}-$ существенные подмодули модуля $M$ и $\alpha^{\prime}: X \rightarrow X^{\prime}-$ изоморфизм. Так как $E-$ инъективный модуль, то найдется эндоморфизм $\alpha$ модуля $E$, совпадающий с $\alpha^{\prime}$ на $X$. Поскольку $X-$ существенный подмодуль в $E$ и

$$
X \cap \operatorname{Ker}(\alpha)=0,
$$

то $\alpha$ - мономорфизм. Поэтому $\alpha(E) \cong E$ и $\alpha(E)-$ инъективный модуль. Тогда $\alpha(E)-$ прямое слагаемое модуля $E$. Кроме того, $\alpha(E)$ содержит подмодуль $\alpha(X)$ и

$$
X^{\prime}=\alpha X \text {. }
$$


Поэтому $\alpha(E)$ - существенное прямое слагаемое модуля $E$. Тогда $\alpha(E)=E$ и $\alpha-$ автоморфизм модуля $E$. Сужение $\alpha$ на $M$ является по условию (1) эндоморфизмом модуля $M$, совпадающим с $\alpha^{\prime}$ на $X$.

Докажем, что (2) $\Rightarrow(1)$. Пусть $\alpha-$ автоморфизм модуля $E$,

$$
\begin{aligned}
X^{\prime} & =M \cap \alpha(M) \subseteq M, \\
X & =\alpha^{-1}\left(X^{\prime}\right) \subseteq M .
\end{aligned}
$$

Так как $\alpha(M)-$ существенный подмодуль модуля $E=\alpha(E)$, то $X^{\prime}-$ существенный подмодуль модуля $M$. Кроме того,

$$
X=\alpha^{-1}\left(X^{\prime}\right)
$$

- существенный подмодуль модуля $E=\alpha^{-1} E$. Так как $\alpha$ индуцирует изоморфизм модуля $X$ на $X^{\prime}$, то по (2) найдется эндоморфизм $\beta$ модуля $M$, совпадающий с $\alpha$ на $X$. Допустим, что $z \in M \cap(\alpha-\beta)(M)$. Тогда

$$
z=(\alpha-\beta)(y)
$$

где $y \in M$. Отсюда

$$
\alpha(y)=\beta(y)-z \in M \text {. }
$$

Поэтому $y \in X$. Но, по построению,

$$
(\alpha-\beta)(y)=z=0 .
$$

Поэтому

$$
M \cap(\alpha-\beta)(M)=0 .
$$

Отсюда получаем, что

$$
(\alpha-\beta)(M)=0,
$$

так как $M-$ существенный подмодуль в $E$. Следовательно, $\alpha(M) \subseteq M$.

Лемма 3. Если $M$ - автоморфизм-инвариантный модуль, то $M$ - автоморфизм-продолжаемый модуль.

Доказательство. Пусть $X$ - подмодуль в $M, \alpha$ - автоморфизм модуля $X$. Надо доказать, что $\alpha$ продолжается до эндоморфизма модуля $M$. По утверждению 2 леммы 1 , без ограничения общности можно считать, что $X-$ существенный подмодуль в $M$. Так как $\alpha$ - изоморфизм существенного подмодуля $X$ автоморфизм-инвариантного модуля $M$ на существенный подмодуль $X$ в $M$, то по лемме $2 \alpha$ продолжается до эндоморфизма модуля $M$.

Если $X$ и $Y-$ модули, и для любого подмодуля $Y^{\prime}$ в $Y$ каждый гомоморфизм $Y^{\prime} \rightarrow X$ продолжается до гомоморфизма $Y \rightarrow X$, то $X$ называется $Y$-инъективным модулем. Для модуля $M$ каждый подмодуль любого фактор-модуля $M$ называется подфактором $M$. В лемме 4 собраны хорошо известные утверждения. 


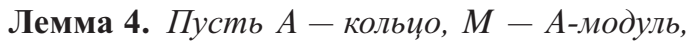

$$
M=\bigoplus_{i \in I} M_{i}
$$

и $X, Y-A$-модули, являющиеся $M_{i}$-инъективньми для всех $i \in I$.

(1) $X \oplus Y-\bar{M}$-инъективный модуль для любого подфактора $\bar{M}$ модуля $M$.

(2) Если для любого $i \in I$ модули $M_{i} u \bigoplus_{j \neq i} M_{j}$ являются $M_{i}$-инъективными, то М - квазиинъективньий модуль.

(3) Каждый модуль, инъективный относительно модуля $A_{A}$, является инъективным модулем.

(4) Если модуль $M$ содержит изоморфную копию модуля $A_{A}$, то модуль $X$ инъективен.

Утверждения 1, 2 и 3 леммы 4 доказаны, например, в [15, 16.1(1),16.2(2)], [9, 1.18] и $[15,16.4]$, а утверждение 4 вытекает из утверждений 1 и 3.

Лемма 5. Пусть $M-$ модуль, $X$ и $Y$ - подмодули в $M$ с условием $X \cap Y=0, u$ $f: Y \rightarrow X-$ гомоморфизм. Тогда существует такой автоморфизм $\alpha$ модуля $X \oplus Y$, что эндоморфизм $\alpha-1$ модуля $X \oplus Y$ совпадает с $f$ на $Y$.

Доказательство. Определим эндоморфизм $\alpha$ модуля $Z$ соотношением

$$
\alpha(x+y)=x+f(y)+y
$$

для всех $x \in X$ и $y \in Y$. Допустим, что

$$
0=\alpha(x+y)=x+f(y)+y, \quad x \in X, \quad y \in Y .
$$

Тогда $\alpha-$ мономорфизм, поскольку

$$
\begin{gathered}
y=-x-f(y) \in X \cap Y=0, \quad f(y)=0, \\
x=x+f(y)+y=\alpha(x+y)=0 .
\end{gathered}
$$

Кроме того, для любых $x \in X$ и $y \in Y$

$$
\begin{aligned}
x+y & =(x-f(y))+(f(y)+y) \\
& =\alpha(x-f(y))+\alpha(y) \in \alpha(X \oplus Y) .
\end{aligned}
$$

Поэтому $\alpha$ - искомый автоморфизм модуля $X \oplus Y$.

Лемма 6 ([1, 3.5]). Если $М$ - прямая сумма равномерных модулей, то М является квазиинъективным модулем в точности тогда, когда $M-$ псевдоинъективный модуль.

Лемма 7. Пусть $A-$ кольц̧о, $M$ - автоморфизм-продолжаемый $A$-модуль, и

$$
M=\bigoplus_{i} M_{i} .
$$

(1) Для любого $i \in I$ модуль $M_{i}$ является автоморфизм-продолжаемым, а модуль $\bigoplus_{j \neq i} M_{j}$ является $M_{i}$-инъективныл. 
(2) Если все модули $M_{i}$ квазиинъективнь, то $M$ - квазиинъективный модуль.

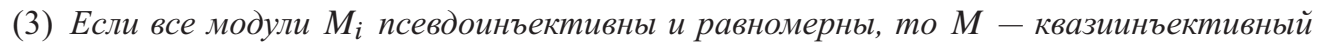
модуль.

Доказательство. Первое утверждение проверяется непосредственно.

Докажем второе утверждение. Обозначим

$$
X=\bigoplus_{j \neq i} M_{j} .
$$

Тогда

$$
M=X \oplus M_{i}
$$

Надо доказать, что $X-M_{i}$-инъективный модуль. Пусть $Y-$ подмодуль модуля $M_{i}$ и $f$ - гомоморфизм из $Y$ в $X$. По лемме 5, существует такой автоморфизм $\alpha$ модуля $X \oplus Y$, что эндоморфизм $\alpha-1$ модуля $X \oplus Y$ совпадает с $f$ на $Y$. По условию, автоморфизм $\alpha$ модуля $X \oplus Y$ продолжается до некоторого эндоморфизма $\beta$ модуля $X \oplus M_{i}=M$. Обозначим через $g$ эндоморфизм $\beta-1_{M}$ модуля $M$. Пусть $u: M_{i} \rightarrow X \oplus M_{i}-$ естественное вложение, и $\pi: X \oplus M_{i} \rightarrow X-$ проекция с ядром $M_{i}$. Тогда гомоморфизм $\pi g u$ из $M_{i}$ в $X$ - продолжение гомоморфизма $f$. Поэтому $X-M_{i}$-инъективный модуль. Так как все модули $M_{i}$ являются $M_{i}$-инъективными, то утверждение 2 следует из утверждения 1 и части 2 леммы 4.

Третье утверждение следует из второго утверждения и леммы 6.

Модуль называется конечномерным, если он не содержит бесконечных прямых сумм ненулевых подмодулей.

Лемма 8 ([12, теорема 17]). Если $M$ - автоморфизм-инвариантныий конечномерный модуль, то $M-$ псевдоинъективный модуль.

Лемма 9. Пусть $M-$ автоморфизм-продолжаемьй модуль.

(1) Если $M$ - артинов модуль, то $M-$ псевдоинъективный модуль.

(2) Если $M$ - прямая сумма артиновых равномерных модулей, то $M-$ квазиинъективный модуль.

Доказательство. Докажем первое утверждение. Пусть $E-$ инъективная оболочка модуля $M, \alpha$ - автоморфизм модуля $E, X$ - сумма всех подмодулей в $M$, переходящих на себя под действием $\alpha$, и $S$ - цоколь модуля $E$. Тогда $S \subseteq M$, поскольку $E-$ существенное расширение модуля $M$. Так как $\alpha(S)=S$, то $S \subseteq X$. Поэтому $X-$ сушественный подмодуль в $E$, поскольку $X$ содержит $S$.

В силу леммы 8 , достаточно доказать, что $\alpha(M) \subseteq M$, иными словами, $X=M$. Допустим, что $X \neq M$. Так как $\alpha(X)=X$ и $M-$ автоморфизм-продолжаемый модуль, найдется такой эндоморфизм $\beta$ модуля $M$, что $(\alpha-\beta)(X)=0$. Пусть $\alpha^{\prime}-$ сужение $\alpha$ на $M, \bar{M}=M / X, \bar{E}=E / X, \bar{\alpha}-$ мономорфизм из $\bar{M}$ в $\bar{E}$, индуцированный гомоморфизмом $\alpha^{\prime}, \bar{\beta}$ - инъективный эндоморфизм модуля $\bar{M}$, индуцированный эндоморфизмом $\beta$ модуля $M$.

Обозначим

$$
\varphi=\alpha^{\prime}-\beta \in \operatorname{Hom}(M, E)
$$


Пусть $\bar{\varphi}$ - гомоморфизм из $\bar{M}$ в $E$, индуцированный гомоморфизмом $\varphi$, и $X^{\prime}-$ полный прообраз в $M$ модуля

$$
X_{1}=X \cap \operatorname{Im} \varphi
$$

при гомоморфизме $\varphi$. Так как $X-$ существенный подмодуль в $E$, то $X_{1}-$ существенный подмодуль в $\operatorname{Im} \varphi$, причем $\varphi(X)=0$. Поэтому

$$
\bar{X}^{\prime}=X^{\prime} / X
$$

- существенный подмодуль ненулевого модуля $\bar{M}$. Тогда артинов модуль $\bar{X}$ содержит ненулевой цоколь $\bar{T}=T / X$ ненулевого модуля $\bar{M}$. Так как $\bar{\varphi}\left(\bar{X}^{\prime}\right)=0$, верно, что

$$
\bar{\alpha}^{\prime}(\bar{T})=\bar{\beta}(\bar{T}) \subseteq \bar{T} .
$$

Поэтому $\alpha(T) \subseteq T$ и $\alpha$ индуцирует мономорфизм артинова модуля $T$ в себя. Тогда $\alpha(T)=T$. Получено противоречие, поскольку $T-$ подмодуль в $M$, строго содержащий $X$.

Докажем второе утверждение. Так как по первому утверждению леммы 7 каждое прямое слагаемое модуля $M$ является автоморфизм-продолжаемым модулем, то по утверждению 3 той же леммы каждое артиново прямое слагаемое модуля $M$ является псевдоинъективным модулем. Поэтому, в силу утверждения 3 леммы $7, M-$ квазиинъективный модуль.

Лемма 10. Если A - артиново справа кольио и $M$ - прямая сумма конечнопорожденных равномерных правых А-модулей, то равносильны условия:

(1) М - автоморфизм-продолжаемый модуль;

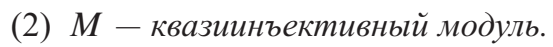

Доказательство. Импликация (2) $\Rightarrow(1)$ проверяется непосредственно.

Докажем, что $(1) \Rightarrow(2)$. Так как каждый конечнопорожденный правый модуль над артиновым справа кольцом является артиновым модулем, то утверждение вытекает из части 2 леммы 9.

Замечание 1. Кольцо $A$ называется кольцом конечного модульного типа, если кольцо $A$ артиново справа и существует только конечное число $n$ неизоморфных неразложимых конечнопорожденных правых $A$-модулей; в этом случае кольцо $A$ артиново слева и существует только $n$ неизоморфных неразложимых конечнопорожденных левых $A$-модулей (см. [2]). Каждый модуль над кольцом конечного модульного типа является прямой суммой конечнопорожденных модулей (см. [13]).

Лемма 11. Пусть $A-$ - кольичо, над которым каждый правый модуль является прямой суммой равномерных модулей.

(1) A- артиново кольцио конечного модульного типа.

(2) Если $M$ - правый A-модуль, то М является автоморфизм-продолжаемыми в точ-

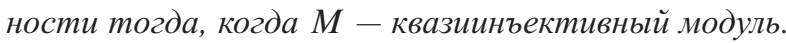

Утверждение 1 леммы 2 вытекает из теоремы 1 в [3], а утверждение 2 вытекает из утверждения 1 , леммы 10 и замечания перед леммой.

Теперь теоремы 1 и 2 вытекают из леммы 11 и утверждения 1 леммы 9 соответственно. 


\section{Список литературы}

1. Alahmadi A., Er N., Jain S. K., Modules which are invariant under monomorphisms of their injective hulls. J.Australian Math. Soc. (2005) 79, №3, 2265-2271.

2. Eisenbud D., Griffith P., The structure of serial rings. Pacific J. Math. (1971) 36, №1, 109-121.

3. Er N., Rings whose modules are direct sums of extending modules. Proc. Amer. Math. Soc. (2009) 137, №7, 2265-2271.

4. Фейс К., Алгебра: кольия, модули и категории, 2. Мир, Москва, 1979.

5. Goodearl K. R., Warfield R. B., An introduction to noncommutative Noetherian rings. Cambridge University Press, Cambridge, 2004.

6. Jain S. K., Singh S., Quasi-injective and pseudo-injective modules. Canadian Math. Bull. (1975) 18, №3, 359-366.

7. Johnson R. E., Wong F. T., Quasi-injective modules and irreducible rings. J. London Math. Soc. (1961) 36, 260-268..

8. Lee T.-K., Zhou Y., Modules which are invariant under automorphisms of their injective hulls. $J$. Algebra Appl. (2013) 12, №2, 1250159.

9. Mohamed S. H., Müller B. J., Continuous and discrete modules. Cambridge University Press, Cambridge, 1990.

10. Singh S., On pseudo-injective modules. Riv. Mat. Univ. Parma (1969) 9, 59-65.

11. Singh S., Jain S. K., On pseudo-injective modules and selfpseudo-injective rings. J. Math. Sci. (1967) 2, 23-31.

12. Singh S., Srivastava A. K., Rings of invariant module type and automorphism-invariant modules. arXiv 1207.5370 [math.RA].

13. Tachikawa H., QF-3 rings and categories of projective modules. J. Algebra (1974) 28, 408-413.

14. Teply M. L., Pseudo-injective modules which are not quasiinjective. Proc. Amer. Math. Soc. (1975) 49, 305-310.

15. Wisbauer R., Foundations of module and ring theory. Gordon \& Breach, Philadelphia, 1991.

Статья поступила 23.10.2012. 